\title{
Measuring External Complexity of Complex Adaptive Systems Using Onicescu's Informational Energy
}

\author{
Vasile Avram \\ Diana Rizescu (Avram) \\ Bucharest University of Economic Studies \\ vasileavram@ie.ase.ro \\ Bucharest University of Economic Studies \\ Diana.Rizescu@csie.ase.ro
}

DOI:10.5901/mjss.2014.v5n22p407

\begin{abstract}
During the last decades the complexity theory of complex adaptive systems has captured the attention of a plethora of people, ranging from scientists and to the general public, through its discussions on emergence, behavior, self-organization, computer simulations and so on with a strong emphasis in the globalization context. The intuitively given explanations about produced phenomena are hardly accepted, by regular people, without giving them scientifically proofs with some measurements and figures. Thus we are forced to find new measurements and analysis tools or to adapt the existing ones, for better understand those systems. The social systems and economic systems become more complex and unpredictable and necessitate the use, among other things, of the acquirements of social psychology to better understand and monitor them. Having a large number of mutually internetworked and interdependent parts the complex adaptive systems are hard to model and understand, so that any theory or practice defined/ applied to reduce the area of the analyzed complexity and making analysis, modeling and simulation feasible is welcome and useful. The analysis of both, external complexity and internal complexity of complex adaptive systems, as defined by Jüergen Jost, become a starting point. The external complexity addresses the volume of inputs (as information/ energy), from system's environment, that the system is capable to manipulate or to process, while, the internal complexity is a measure of input representation by the system, in fact, the model complexity. For both categories the existing model uses entropy, or other formulas based on, to measure the system. This paper introduces the usage of Onicescu's Informational Energy theory and measures, based on frequencies used in statistics, to approximate the measurements of external complexity. The aim of the paper is not to use a technology instead another, but a way to simplify and express some measurements in terms of normal frequencies, the general people encounter daily and better understand, and not in terms of probabilities that requires mathematics skills and, maybe, sophisticated computational tools from the normal reader part, for better understanding.
\end{abstract}

Keywords: External Complexity, Complex Adaptive Systems, Informational Energy, Entropy

\section{Introduction}

The presentation in this paper was oriented to an easy formal style and, for that reason, the introduced concepts have a surname given as a single letter used later on to define mathematical models and their computational formulas. The systems can be broadly defined (Avram\&Dodescu, 2003) as sets of functionally inter-correlated elements (E) that acts to achieve specific goals (objectives, one or more). The elements are described by properties and behavior, specific and/ or common to all. Systems are combinations of elements including all or some of the sources: objectives $(\mathrm{Z})$, inputs $(\mathrm{X})$, outputs $(\mathrm{Y})$, processes $(\mathrm{P})$, and other internal relationships (dominant; collaboration; prediction), and a boundary with the rest of the universe, or system's environment. The way the system reacts to changes (generally on inputs) forms the system behavior. The systems are artifacts created by humans to facilitate communication, abstraction and, analysis and they simplify the explanation of phenomena that appears complex and unrelated. If the artificial systems are fruits of human thinking, the natural ones have an objective existence (they exists independently of human concern, as the solar system, for example). A system is characterized by its status (S) dependent, as a rule, on inputs and represented by any well-defined conditions or properties that can be recognized as such if again appear. If the status of the system changes the system categorized as dynamic, otherwise as static. A system is said adaptive if depending on its status and the status of its environment can 
act differently to attain its objectives or even can change those objectives. If a system realizes exchanges, as inputs and outputs, with its environment, the system is open, as opposite for lack of those exchanges, when closed. The elements of the system are the smallest parts that can be used in system description and modeling. An element belongs to a system if and only if it contribute to the system's activity. The elements can form distinguishable parts to the system, and these, in turn, can participate to form larger (aggregated) parts (parts from parts, recursively) to the system. The elements and parts are coupled together to form a whole. If the parts inside system have the characteristics of a system in fact they are systems in their own right and they are called subsystems. Each element influences the elements to which coupled by influencing the input of those elements or, generally, the environment of those elements. The complex systems have a large number of mutually internetworked and interdependent parts and, at least for this, are hard to model and understand. Formally, two elements (or parts) e1 and e2 can be coupled in one of the ways:

- e1 is coupled with e2 so that it influence e2 and e2 has no influence to e1. We can say that e1 is dominating e2 (is a normal unidirectional communication channel or is a feed-forward/ prediction link);

- e1 is coupled to e2 so that both coupled elements reciprocally influenced. We can say that between e1 and e2 we have a feedback (the value of this can be positive or negative and the result is called collaboration).

\subsection{Complex Systems}

"Complex systems consist of many interacting components and many hierarchical layer and, in general, it is impossible to reduce the overall behavior of the system to a set of properties characterizing the individual components. The interaction between components is able to produce properties at the collective level that are simply not present at the component considered individually. (Moffat, 2003)" Heylighen shows in (Heylighen, 2008) that the complex systems "components are in the same time distinct and connected, both autonomous and to some degree mutually dependent". In simple words it means that the properties and behaviors at the whole system level (its higher hierarchical level) are not a summation of properties and behaviors at lower, or generally base level. In a hierarchical organization of the system, each layer, at a higher level in the hierarchy can have properties and behaviors unfound at lower levels of its base hierarchy.

Between coupled elements take place communications, of different natures, that send (outputs) and receive (inputs) messages or signals containing messages. In simulations and models of the systems we can focus our research and analysis on that type of communication but we must have in mind the strong link with (or determination of) the material or energy flux taking place in parallel or sequentially, on the same channel. The system achieves its objectives by the process of converting inputs (information, transactions data, raw material, parts, electrical pulses, light, water and minerals etc.), that supplied by its environment and crosses the boundaries to become parts of the system, into outputs (decision and control information, finished goods, parts, or any other kind of output such as carbon dioxide, urine and feces, common for living organisms) that crosses the systems boundaries to become parts of the environment. Not all kinds of elements of a system must be present to a concrete one, for example, inputs and outputs are missing for closed systems (they do not exchange any kind of inputs and outputs with their environment).

"The social systems are different in many aspects of most other kinds of systems in the way the elements belong to the system or not. The system do not "die" if one (or some) of its members eliminated from the system. The social systems implies individuals and their relationships to form groups and to interact with other individuals, groups, and institutions. As members of different forms of social organization they obey to laws and rules of that. The individuals can participate in the same time in many different groups and generally can quit a group by different reasons (can migrate in another group, can die etc.). In such systems applied the large numbers low: we can eliminate/ add individuals/ groups from/ to the system without affecting the whole system. Consequently the subsystems of social systems are very hard to be precisely delimited by the nature of their boundaries. Thus, the social systems become more complex and unpredictable and necessitate the use of social psychology to better understand and monitor them. (Rizescu\&Avram, 2013)"

\subsection{Complex Adaptive Systems}

As starting point, here are accepted the complexity sources and characteristics as outlined by Fromm (2004, p. 23): "Complexity emerges from the clash of opposite forces, and is characterized by consonance in dissonance, regularity in irregularity, cooperation in separation, integration in differentiation, order in chaos, simplicity in intricacy and unity in diversity". The complexity is considered as being situated somewhere between order and disorder and the complex systems are not neither, regular and predictable and, nor random and chaotic (Heylighen, 2008). The complexity of a system is the 
amount of information needed in order to describe it which, in turn, depends on the level of detail required in its description (Bar-Yam, 1997).

The complex adaptive systems (CAS) are dynamic systems able to adapt and change within, or as part of, a changing environment (Moffat, 2003). The components of complex systems are most commonly modeled as agents (for example, people, firms, animals, cells and molecules, neurons etc.), usually are goal-directed (try to maximize individual fitness, utility or, preference) or, if not goal-directed follows a cause-and-effect or condition-action logic, their number is variable (they can multiply or "die" within the system) and, the environmental conditions to which an agent reacts can be affected by other agents (Heyligen, 2008). The complex adaptive systems have three basic characteristics: adaptation, self-organizing and emergence (Heyligen, 2008). The self-organizing is a characteristic given by the system's structure, by its internal processes and, represents the tendency of the larger dynamic systems to organize them itself in a critical status of high organization (perfection). The emergence (from Latin "emergere") defines a qualitative change in the system behavior when changing its observation scale, such as from one element scale to group of elements scale. Emergence is a common characteristic of complex adaptive systems for which the behavior of the whole is more complex than the sum of behaviors of its elements and parts. The emergent properties have two important aspects: they lose when decompose the system in its component parts and, when a component is eliminated (detached) from the whole this one loses its emergent properties. We can say that the system (the properties and functionalities of) is always more than the sum of its parts.

The study of complex systems and their parts, of interaction among them and of behavior, cannot be always done on the system itself. The common scientific way is to ignore some system details and to find a higher level abstraction of the system as a model that can permit realizing simulations of the studied system. Miller and Page defines in (Miller\&Page, 2007) a general model (or modeling) of a system characterized by a set $S$ of different statuses and a transition function (a collection of processes, procedures, functions) $F(S)$ that maps a given status at moment $t$ into a new status at moment $t+1$. If the dimension of status space is too high this one can be reduced by generating few equivalence classes (mapping) $E(S)$ which maps a subset of real world statuses $S$ into a model status $s$ and, in this context, finding a transition function $\mathrm{f}(\mathrm{s})$ useful for modeling. In a cybernetic context (here introduced as described in Avram\&Dodescu, 2003), the inputs $X$ are transformed by processes $P$ inside of the system to obtain the results (outputs) $Y$ and we can express that by a generic equation of the system that can be written as $Y=P(X)$. Most of the time between the outputs and objectives we have some differences that can be expressed as Z\#Y. For that reason, it is necessary to correct this differences by intermediate of a regulator $\mathrm{R}$ (a feedback processor) that corrects the inputs by an average value $\Delta \mathrm{X}$ (that can be positive or negative feedback) and the generic equation can be written as: $Y=P(X+\Delta X)$. This describes in a simple way an adaptive system.

The general approach in studying and simulating on computers of complex adaptive systems is realized by iterating some simple rules locally in an interacting agent population which generate in turn complex global phenomena. The usual representation of elements and parts of a complex system together with their internal relationships (and dependencies) is in a shape of complex networks. Examples of complex networks are neuronal networks, social systems, social networks, neural system, economic systems etc.

\section{Methodology}

Initially the developments and conformity checks realized on some dependant characteristics about Internet infrastructure represented by the server operating systems and webserver software used. The results are very satisfactory from many points of view, but, since data collected and based only on online surveys the proof of the validity of proposed formulas for the measurements must be realized on an incontestable trustable source. To find the circumstances and ways to replace the difficult computations, such as conditional probabilities, here used the statistics data obtained by 2011 census in Romania (Romania Census, 2014) and selected the repartition of population on age categories, genders and area and, adapted as notations to the needs of the mathematical models, as shown in Table 1. The columns in the table represents the events categories and they are mutually exclusive. The data inside table refers to (calculated based on) grand totals at country level. For columns and rows used letter characters as an alias of that used, later on, to express the computational models and to evaluate them, for conformity checks of the theoretical findings.

Table 2 is a sample randomly selected (by generating of two random numbers, one indicating the age and the other the number of the detail line for area) taken from similar data in the 2010 Romanian Statistics Anuarry (2011:1) at detail level (population on age groups, counties, cities and, gender). The sample (excerpt) is shown in Table 2. The total population was taken 1000 for the sake of simplicity in mental computations and is not computed as for statistical surveys. When we 
want to make a specific research, for a specific target population, these aspects will have their own importance and accuracy, as required.

Tables 1 and 2 used to show how to compute both, entropy and informational energy on data refering to total population (Table 1) or to samples from that population. If you compare the tables you see that the probabilities associated to corresponding categories are closed while between the corresponding absolute value do not exist a vizible common rule (such as division, multiplication or other kind of formula) to determine the excerpt value from its corresponding total value, this poofing the validity of the selection realized randomly.

Table 3 contains processed from data taken from the 2011 Romanian Statistics Anuarry (2011:1, 2011:2), Chapter 2 Population and Chapter 3 Labour Market. It shows the structure, as absolute values and frequencies/ probabilities, of two broad categories of population defined depending on their participation on the labour market mainly, active population and non economically active persons, distributed by group ages. For the active persons are indicated the common characteristics for different input model structure in the Labour Market (sub)system, by $\{\gamma 1, \gamma 2, \gamma 3, \gamma 4, \gamma 5, \gamma 6\}$, as designators for the age categories. These characteristics are common for every reporting year.

Table 4 shows the retirement age combinations, for both sexes, male and females, used in Romania after 2001. These combinations affects the $\gamma 5$ and $\gamma 6$ categories in Table 3 , so we consider having different input models for labour market, denoted in the table by the column $\theta$ (for which all entries having status "current" covered by ninth entry in table).

The tables and the evaluations of different formulas are realized in Excel 2013 and imported in this document.

\section{Representing System Statuses as Weights}

Every kind of system, either natural or artificial, is individualized by some qualitative and/ or quantitative characteristics related to their inputs, outputs, internal relationships etc., defining its status. The status, when represented by energy or information, can be revealed by related weights or ratios. The most common way to determine that ratios or weights is as frequencies or as probabilities. Consider a system characterized by the statuses $s 1, s 2, \ldots$, sn having the corresponding associated weights $\mathrm{w} 1, \mathrm{w} 2, \ldots$, wn with respect for the constraint $\sum_{i=1}^{n} w_{i}=1$. In Table 1 the statuses denoted by yi (they are the si equivalents, the notation in table having importance for the correlation coefficient of Informational Energy and for extended input of external complexity) are represented in absolute value on column "Total Population" (or T) and as and as frequencies (in that case as probabilities too since computed on entire population) in column wi. Both, probability and frequency, uses a similar formula, and we can say they are the mathematics of chance. The difference is that they address to different sets of population (or different scales): the probability request measurements on the entire (whole) population and, the frequency request only representative excerpt (or sample) from the population (a small percent from total population). If sk is an event (or status) that can occur in a population having $n$ members $(k=1,2, \ldots, n)$ then the frequency (weight, in the context) is $w_{k}=\frac{\text { frequency of } s_{k}}{\text { sum of frequencies }}$ with respect of constraint $\sum_{i=1}^{n} w_{i}=1$. For our case in Table 1 column 2 these are $\{0.0505,0.0491,0.0516,0.0563,0.0803,0.0752,0.0827,0.0776,0.0811,0.0566,0.0688,0.067,0.054$, $0.041,0.0435,0.0331,0.0206,0.0109\}$ and their sum is 1 .

For the entire population (or statuses) the frequency of sk is exactly the number of times sk occurs within the population over the sum of frequencies that is the total number of population $n$ and the frequencies equals the probabilities. It is intuitively clear that observing and measuring excerpts of a population is easy than do the same for the entire population.

All the measurements realized are helpful, from a point of view, to understand the system status and maybe to predict its next behavior. Not all the time, the measurement of the different statuses taken in isolation, are helpful in understanding the observed phenomena. In real world contexts and systems the statuses depends one of another due to the multitude of relationships between the system's elements that they characterizing. Thus if we want to measure or to know the probability of a status sk, in correlation with all its influencing statuses to which related, the computation formula for the probability is changed. We must compute the so called conditional probability: it means the probability of the random variable sk conditioned of the knowledge of all other random variables on which depends. In other words, to compute the chance of a status, we must know the chance of all other related statuses. We give down here a very simple expression of computation formula as given in (Beisbart\&Hartmann, 2011). Let's $S$ and T two events for which we assume that the probability of $T$ is non-zero, $P(T) \neq 0$. The conditional probability of $S$ given $T$, denoted by $P(S \mid T)$ is the probability of the joint occurrence of $S$ and $\mathrm{T}$ (it means $\mathrm{S} \cap \mathrm{T}$ - what they have in common), divided by the probability of $\mathrm{T}, P(S \mid T)=\frac{P(S \cap T)}{P(T)}$ and, the probability 
of the joint occurrence of $\mathrm{S}$ and $\mathrm{T}$ is $P(S \cap T)=P(T) \times P(S \mid T)$. The columns 4 (S, Female) and $6(\mathrm{U}, \mathrm{Male})$ from Table 1 are computed as conditional probabilities relatively to total population.

The complexity of the measurements is in direct ratio with the number of conditionings and becomes harder determined as increases the number of the conditionings. The largest number of dependencies of one characteristic or category on the other characteristics or categories of a system is a specific of the social systems. Concluding, as the number of dependent analyzed characteristics increases, the difficulty in determining them increases too (we can say in an exponential way for the human mind, alone).

In this context the internal probability distribution $P(X(\theta))$ used to quantify the informational value of data in the Jüergen Jost model of internal and external complexity, is in most of the cases a conditional probability. This is given by the intrinsic dependencies between the inputs (modeled as data representing the volume, the amount) from the environment, each pattern of that being an expression of dependencies in a specific context (such as a time scale, for example), corroborated with the internal complexity as complexity of representation of that data. This can be argued by analyzing the $\theta$ input models taken as example in Table 4. The input for the labour market is represented by the same categories, generically named yi in Table 3, but we increase the input amount when we change the retire age for womens, it means we change the upper bound of the last category. When the age is not the same, for Male and Female, we are forced to compute conditional probabilities and to work on a joint data set between the sets associated to each sex, as shown in Table 1 columns $S$ and $\mathrm{U}$. When the age is the same for both genders we can work at a centralized level (as shown in Table 3, column "Total Population") and we reduce the inputs complexity and increase in the same time the amount taken on one input source, it means in the scope of the external complexity model.

\section{External Complexity and Internal Complexity}

The complex adaptive systems modeled as networks consists of intelligent autonomous agents (as nodes or constituents of parts). By definition an agent collect information about its environment and applies some methods to select a properly action. According to Fromm $(2004)$ based on Holland $(1995,1997)$ the complex adaptive system (CAS) can be defined as a collectivity of self-similar adaptive agents in interaction and, the multiple-agents system (MAS) as a system composed by multiple agents in interaction. The notions of MAS and CAS are similar, but only apparently and that because they reflect different components of complex systems. CAS is associated with the higher level structures and phenomenon of the system while MAS is associated with the components and elements of base level of that. A complex adaptive system is nothing else than a system that can be simulated, described and explained by a dynamic multi-agent system, and reciprocally, the multi-agent system simulations can be described as complex adaptive systems.

The system's environment is always more complex than the system itself (Jost, 2005) and, consequently never cannot be completely predicted. The system depends on environment regularities in order to maintain its replenishment with the required energy necessary to support (or preserve) its internal structures and processes. CAS try to reduce the internal complexity and to increase the external complexity (Jost, 2005). Jüergen Jost defines in (Jost, 2005), both, external complexity and internal complexity and found a way to model them, as summarized in this paragraph. The external complexity is defined as a measure of the input volume (information and/ or energy measurable by entropy) obtained from the environment and that the system is capable to manage and process: given a model $\theta$ the system can model data as $X(\theta)$, with $X=(X 1, X 2, \ldots, X k)$ the set of input types. $X(\theta)$ introduces an internal probability distribution $P(X(\theta))$, to quantify the informational value of data, so that we can measure its organization using the entropy formula:

$-\sum_{i=1}^{k} P\left(X_{i}(\theta)\right) \log _{2} P\left(X_{i}(\theta)\right)$

in the hypothesis that the system will try to maximize its external complexity. The probability distribution is associated in fact to the partial knowledge about an out from equilibrium system. The system is considered in the equilibrium state when all probabilitie of its statuse where equal. Entropy is maximum for equal probabilities, is unaffected by extra states of zero prbability and, changes for conditional probabilities computed on the same original dataset distributed over dependant characteristics (like total population distributed on age categories and that distributed on sexes, Male and Female, as shown in Table 1 and Table 2). When computed on messages the entropy measure our ignorance about the message in its entirety or, in other words, "how likely the best predictions of the rest of message are to be wrong (Sethna, 2006, p. 88)". The entropy calculated here on data in Table 1 for the dataset in column labeled 2 and is 4.1699 which indicate a highly predictable dataset and is aproximatively 6 times less (read less organized) than maximum entropy 24.2622. When we express the dataset on two broad categories represented by sexes the conditional probabilities associated to age categories are distributed on columns 4 and 6 . The entropy computed on these values is 5.0748 , increased relatively to the one 
computed on column 2 but in accordance with its behavior "entropy changes with conditional probabilities". The entropy now is only approximatively 5 times less than the maximum entropy, we can interpret the measured system as a bit more organized. The entropy is now computed on the sample data in Table 2 column 2 and is 4.0627 which indicates, as the one determined for Table 1 column 2, a high predictable dataset. Until now the computations of entropy realized with the scope to have enough examples for comparissons with the informational energy, later on. The inputs for the labour market, in Table 3, designated by $\{\gamma 1, \gamma 2, \gamma 3, \gamma 4, \gamma 5$, $ү 6\}$, it means the values $\{0.0889,0.2677,0.2967,0.1946,0.1126,0.0395\}$, forms a complete system of probabilities and corresponds to nineth $\theta$ model in Table $4,\{65,65\}$. In this context the valuation of the formula is 2.3379 which is approximatively 10 times less than the maximum entropy of considered population (14, 946, 046), it means 10 times less organized than maximum possible (23.8333). This is very good since a low entropy dataset is highly predictable. Thus the internal complexity is defined as a measure of the complexity of the representation of the input by the system (representing in fact the model complexity). When external complexity measured as entropy this can be viewed as data complexity. The scope of the system is to manage as many inputs as possible using the simplest possible model. The increasing of external complexity is dependent on the time scale involved and the system is considered that has gathered input, noted with $\Xi$, on a time scale when the distribution of input patterns become stationary. After attaining that stationary state the model should be improved to handle as many inputs as possible, which is defined by Jost with formula:

$-\sum_{i=1}^{k} P\left(\Xi_{i} \mid \theta\right) \log _{2} P\left(\Xi_{i} \mid \theta\right)-\log _{2} P(\theta)$

where $P(\theta)$ is the probability assigned to the the model (dependent on its internal structure). The $\Xi=\{0.0410,0.1236$, $0.1370,0.0899,0.0520,0.0183,0.1505,0.2569,0.0909,0.0335,0.0277,0.0311,0.0733,0.1314\}$, build by extending the input to the entire population able to participate on labour market (is excluded the category of childrens and the part of retired or too old from the last category). The formula here evaluates to $3.4438-(-1.1148)=4.5586$ and here is an improvment since is only 6 time less than the maximum entropy (28.3839) in that case when population on input increses $(18,141,057)$. The determination of this improvment of the system must be realized while minimizing its internal complexity, defined as:

$$
\min _{\theta}\left(\log _{2} P\left(\Xi_{\mathrm{i}} \mid \theta\right)-\log _{2} \mathrm{P}(\theta)\right)
$$

an objective function like for the model. The objective function of the model try to minimize the difference between how efficient is the model (the first term) and how complicated is the model (the second term). So, can be considered that the external complexity addresses the volume of inputs (as information/ energy), from system's environment, that the system is capable to manipulate or to process, while, the internal complexity is a measure of input representation by the system, in fact, the model complexity. For both categories the existing model uses entropy, or other formulas based on, to measure the system.

\section{Onicescu's Informational Energy}

This paragraph is based on the information provided by Onicescu itself in the reference (Onicescu\&Stefanescu, 1979) and the presentation in reference (Rizescu\&Avram, 2014). The concept of informational energy and associated theory and practical methods developed by Onicescu before the year 1979.

Consider a system characterized by the statuses $\mathrm{s} 1, \mathrm{~s} 2, \ldots$, sn having the corresponding weights $\mathrm{w} 1, \mathrm{w} 2, \ldots$, wn with respect for the constraint $\sum_{\mathrm{i}=1}^{\mathrm{n}} \mathrm{w}_{\mathrm{i}}=1$. The global information of the system, denoted by $\mathrm{ES}$, can be expressed by the Onicescu "Informational Energy", determined as the mean of weights wi relatively to the individual statuses si, by the formula:

$$
\mathrm{E}_{\mathrm{S}}=\sum_{\mathrm{i}=1}^{\mathrm{n}} \mathrm{w}_{\mathrm{i}}^{2}
$$

(a sum of squares of weights), with $\frac{1}{n} \leq E_{S} \leq 1$.

The informational energy is $1 / n$ only when all statuses have the same weight $1 / n$ (the system's uniformity attained) and is 1 when one of its statuses weight is 1 (and consequently all others 0 ). For Table 1 the Informational Energy in column 2 is 0.0629 which indicate a relative differentiation from equal chance case (0.0073), and is approximatively $1,265,424$ times far away of maximum (1). To the same data corresponds an entropy of 4.1699 which indicates a relative differentiation from perfect organization and compared with the maximum entropy of 24.2622 which is 6 times greater. So, both measurements, given to us a similar characterization of the system which is not in its equilibrium state, is not uniform, and not completely disorganized. The informational energy computed now on the conditional probabilities in Table 1 (columns 4 and 6 ) this is 0.1237 , it increases relatively to the one computed on data in column 2 . The increase means that the indetermination, 
uniformity or, disorganization of the system decreases (is a little bit more organized), a similar conclusion as for corresponding entropies. The informational energy is now computed on the sample data in Table 2 column 2 and is 0.0628 which is closed to the one determined for Table 1 column 2 and indicates a high predictable dataset too. Based on comparissons realized between entropy and informational energy we can conclude that the both measurements gives to us comparable information about the system. For Table 2 column 4 and 6 we compute the informational energy values for each one because they are individual complete probability repartitions (the sum of the values in each one is 1).

It was proved that the informational energy decreases in direct proportion with the raising of the systems uniformity, disorder, or indetermination. In other words, the informational energy increases in direct proportion with the raise of system differentiation that is the source of social system existence, for example. The informational energy of a system composed by two or more independent elements (or parts) is the product of their corresponding informational energies. For social systems (or groups of people or, more formally, agents) and in the context of globalization is difficult to find, in the same space, two populations having nothing in common. Thus is possible to determine the correlation between two population (or groups of, or generally parts of a system) $S$ and $T$, noted $C S, T$, having in common the characteristics $\gamma 1, \gamma 2, \ldots, y n$ with the corresponding weights w1, w2, .., wn, for population $\mathrm{S}$ and, q1, q2, .., qn for population $\mathrm{T}$, using the formula:

$\mathrm{C}_{\mathrm{S}, \mathrm{T}}=\sum_{\mathrm{i}=1}^{\mathrm{n}} \mathrm{w}_{\mathrm{i}} \cdot \mathrm{q}_{\mathrm{i}}$

The formula (5) permit us to have dependent sets we associate complete sets of probabilities, it means without computing first the conditional probabilities, and to see if between two population is a relationship (like having something in common). The CS, T evaluates to zero (CS, $T=0$ ) if and only if all terms in the expression are null (the two parts or populations do not have something in common). The correlation coefficient is applied for data in columns 4 and 6 in Table 2, as separate datasets, and its value 0.0624 is closed to the informational energy for the entire sample 0.0628 . The value of the correlation of a population $S$ with itself is his informational energy. The informational correlation coefficient (noted RS, T ) of the populations $\mathrm{S}$ and $\mathrm{T}$ obtained by normalization of the correlation:

$$
\mathrm{R}_{\mathrm{S}, \mathrm{T}}=\frac{\mathrm{C}_{\mathrm{S}, \mathrm{T}}}{\sqrt{\mathrm{E}_{\mathrm{S}} \cdot \mathrm{E}_{\mathrm{T}}}}
$$

where RS, $T=1$ if and only if $S$ and $T$ identical. The correlation and the correlation coefficient can be applied to any number of population having in common the characteristics $\gamma_{1}, \gamma_{2}, \ldots$, , $\mathrm{n}$ by extending the formulas with the corresponding weights.

\section{Conclusions and Discutions}

When we make use of complex adaptive systems and use entropy to measure and characterize the external complexity we must determine the conditional probabilities, and we can say that this is very hard be realized when the conditioning involves more than two conditioning input statuses. If we have the complete probability system then we can apply the computation formula and see the difference from maximum entropy. The frequencies, determined for the inputs from environment, are used as source for informational energy and correlation coefficient determination. Thus, instead computing the conditional probabilities is easy to determine the frequencies input status and to compute the informational energy and the correlation coefficient.

The - (minus) sign in the formulas (1), (2) and (3) is required to produce a positive value since the logarithm on fraction numbers (as probabilities are) produces a negative value. Because for informational energy we use square values all results are positive. With these considerations, shown here and in the previous paragraphs, the original model of external complexity, described by the equations (1), (2) and (3), based on entropy, can be adapted to use informational energy, by the formulas:

$$
\begin{aligned}
& \sum_{\mathrm{i}=1}^{\mathrm{k}} \mathrm{P}\left(\mathrm{X}_{\mathrm{i}}(\theta)\right)^{\wedge} 2 \\
& \sum_{\mathrm{i}=1}^{\mathrm{k}} \mathrm{P}\left(\Xi_{\mathrm{i}} \mid \theta\right)^{\wedge} 2+\mathrm{P}(\theta)^{\wedge} 2 \\
& \min _{\theta}\left(\mathrm{P}(\theta)^{\wedge} 2-\mathrm{P}\left(\Xi_{\mathrm{i}} \mid \theta\right)^{\wedge} 2\right)
\end{aligned}
$$

In the last formula (3') the terms are changed between them because in (3) the first term produces a negative value while the second a positive one, the operation is to minimize the difference between them. For entropies on excerpts the distance between the entropy and the maximum entropy is uncontrolable reduced. For our example in Table 2 column 2 the maximum entropy is only 9.9657 that is too less than the maxim entropy 24.2622 in Table 1 column 2, so we can say the entropy for the sample is not a very good measurement. We can say that for samples, because the number $n$ of counted 
events is drastically reduced, the maximum entropy computed for $1 / \mathrm{n}$ is reduced too and this will influence in direct proportion, we can say, our conclusions. If the entropy is closed on that maximum we can conclude that the equilibrium of the system attained and we must do something to change that status because it becomes impredictible. In reality our system can be highly predictible.

For the same data the informational energies are very closed, 0.0629 and 0.0628 respectively, and they have a comparable distance against the minimum (1/n) and maximum informational energy value 1 . The minimal informational energy in tabe is calculated for equal chance associated to measured categories and is the same for both tables $(0.0556)$. The problem, when we use a sample with a reduced counted people the ratio $1 / n$ increases (relatively to the number of entire population) and in this situation we can conclude that our computed value is to closed on the lower bound and the system is highly predictible, in reality, for the entire population, being far away of that. When energy is closed to lower limit we consider the system as disorganized, undetermined and uniform and that situation can make us searching for new ways to change that. The outlined characteristics shows in fact that informational energy uses as source of data the normal statistical measurements used to characterize the evolution of a society, an economy, a group of countries etc. That is, as the large numbers law stipulates, we can measure only representative excerpts without affecting the real understanding of the behavior. It can be directly applied to measure, similarly to entropy, the disorder degree of a system together with the degree of interdependencies, of its observed parts, by intermediate of the coefficient of correlation.

The informational energy measurements don't exclude or completely replace the usage of entropy and valuation of that for a given system. They can be used together and valuated on the available data source on an efficiency basis and in correlation with the dataset characterizing the observed system. What we can say is that the informational energy can be used for both cases, those of entire population and those of representative sample because a higher lower limit is not so bad (we have a higher energy in reality while mesurements indicates a bit less). The usage of entropy for such samples change the maximum value of that and we can conclude that a system is highly organized and nothing to improve. In this context we can say that the impediments of each one on excerpts can be avoided if we know the total number of population $n$ and we use $1 / n$ in the computation of maximal entropy and as lower limit for informational energy. This replacement allows to increase the accuracy of measurements and the trust in these.

\section{References}

Jost, J. (2004). External and internal complexity of complex adaptive systems. Theory in Biosciences Volume 123, Issue 1,25 June $2004,69-88$.

Onicescu, O., \& Stefanescu, V. (1979). Elemente de statistica informationala cu aplicatii/ Elements of informational statistics with applications. Editura Tehnica, 11-31.

Rizescu, D., Avram, V. (2013). Using Onicescu's Informational Energy to Approximate Social Entropy. Procedia - Social and Behavioral Sciences, Volume 114, 21 February 2014, ISSN 1877-0428, 377-381.

Beisbart, C., \& Hartmann, S. (2011). Introduction. In Beisbart, C., \& Hartmann, S. (Eds), Probabilities in Physics, Oxford University Press, 1-12.

Bar-Yam, Y. (1997). Dynamics of Complex Systems. Addison Wesley.

Fromm, J. (2004). The Emergence of Complexity, Kassel University Press, ISBN 3-89958-069-9, Kassel, Germany.

Holland, J. (1995). Hidden Order, How Adaptation Builds Complexity, Helix Books, Addison-Wesley Publishing Company.

Heylighen, F. (2008). Complexity and self-organization. In Bates, M., Maack, M.N. eds. Encyclopedia of Library and Information Sciences, Third Edition, Taylor\&Francis.

Holland, J. (1997). Emergence, Philosophica, nr. 59, 11-40.

Bailey, K. (1997). System entropy analysis. Kybernetes, 674-687, Volume 26 issue 6/7, ISSN: 0368-492X.

Bailey, K. (2005). Beyond System Internals: Expanding the Scope of Living SystemsTheory, Systems Research and Behavioral Science 22 (6), 497-508.

Bailey, K. (2008). Boundary Maintenance in Living Systems Theory and Social Entropy Theory, Systems Research and Behavioral Science 25, 587-597. 
Miller, J., Page, S. (2007). Complex Adaptive Systems: An Introduction to Computational Models of Social Life. Princeton University Press, Princeton and Oxford.

Moffat, J. (2003). Complexity Theory and Network Centric Warefare. CCRP Publication Series.

Swanson, G. A., \& Bailey, K. (2006). Social Entropy Theory, Macro Accounting, and Entropy Related Measures. In Proceedings of the Annual Meeting of the International Society for the Systems Sciences. Sonoma.

Avram, V., \& Dodescu, G. (2003). Informatics: Computer Hardware and Programming in Visual Basic. Editura Economică, Bucureşti, 18-40.

Sethna, J. (2006). Statistical Mechanics: Entropy, Order Parameters, and Complexity. Oxford University Press 2006 v1.0, 77-104.

Romania Census (2014). Romania Census 2012: Populatia stabila pe sexe si grupe de varsta - categorii de localitati, macroregiuni, regiuni de dezvoltare si judete/ Resident population by sex and age group - categories of localities, macrodevelopment regions and counties. National Institute for Statistics. http://www.recensamantromania.ro/rezultate-2/.

Statistics Anuarry (2010). Romania Statistics Anuarry 2010: Chapter 2 Population. National Institute for Statistics. http://www.insse.ro/cms/files/Anuar\%20arhive/serii\%20de\%20date/2010/pfd/ASR\%202010_romana.pdf, ISSN: 12203246, 35-80.

Statistics Anuarry (2011). Romania Statistics Anuarry 2011: Chapter 2 Population. National Institute for Statistics. http://www.insse.ro/cms/files/Anuar\%20arhive/serii\%20de\%20date/2011/pdf/ASR\%202011_romana.pdf, ISSN: 12203246, 44-78.

Statistics Anuarry (2011). Romania Statistics Anuarry 2011: Chapter 3 Labour Market . National Institute for Statistics. http://www.insse.ro/cms/files/Anuar\%20arhive/serii\%20de\%20date/2011/pdf/ASR\%202011_romana.pdf, ISSN: 1220$3246,117-129$.

Tables

Table 1. The Probabilities Associated to System's Characteristics yi and the Conditional Probabilities for S and U Parts of the System for the Same Characteristics (2011 Romania Census)

\begin{tabular}{|c|c|c|c|c|c|c|c|}
\hline \multirow{2}{*}{\multicolumn{2}{|c|}{$\begin{array}{l}\text { Characteristics } \\
\text { (Age Category) }\end{array}$}} & \multicolumn{2}{|c|}{ T (Total Population) } & \multirow[t]{2}{*}{ S (Female) } & $\begin{array}{l}\text { Probability of } \\
\text { S conditioned }\end{array}$ & \multirow[t]{2}{*}{ U (Male) } & $\begin{array}{l}\text { Probability of } \\
\text { U conditioned }\end{array}$ \\
\hline & & & $\{w i\}$ & & By $T \quad\{$ pi $\}$ & & By $T \quad\{q i\}$ \\
\hline 0 & & 1 & 2 & 3 & 4 & 5 & 6 \\
\hline Y1 & $0-4$ & 1045029 & 0.0505 & 507949 & 0.0252 & 537,080 & 0.0267 \\
\hline y2 & $5-9$ & 1054391 & 0.0491 & 512532 & 0.0255 & 541,859 & 0.0269 \\
\hline y3 & $10-14$ & 1090226 & 0.0516 & 530854 & 0.0264 & 559,372 & 0.0278 \\
\hline Y4 & $15-19$ & 1108453 & 0.0563 & 540381 & 0.0269 & 568,072 & 0.0282 \\
\hline ү5 & $20-24$ & 1366374 & 0.0803 & 662022 & 0.0329 & 704,352 & 0.0350 \\
\hline y6 & $25-29$ & 1303077 & 0.0752 & 636401 & 0.0316 & 666,676 & 0.0331 \\
\hline Y7 & $30-34$ & 1522719 & 0.0827 & 749707 & 0.0373 & 773,012 & 0.0384 \\
\hline y8 & $35-39$ & 1538897 & 0.0776 & 756054 & 0.0376 & 782,843 & 0.0389 \\
\hline ү9 & $40-44$ & 1743878 & 0.0811 & 856309 & 0.0426 & 887,569 & 0.0441 \\
\hline $\begin{array}{l}\text { Y1 } \\
0\end{array}$ & $45-49$ & 1076258 & 0.0566 & 531963 & 0.0264 & 544,295 & 0.0271 \\
\hline $\begin{array}{l}\mathrm{y} 1 \\
1\end{array}$ & $50-54$ & 1332266 & 0.0688 & 677525 & 0.0337 & 654,741 & 0.0325 \\
\hline
\end{tabular}




\begin{tabular}{|c|c|c|c|c|c|c|c|}
\hline $\begin{array}{l}\text { y1 } \\
3\end{array}$ & $55-59$ & 1448043 & 0.067 & 759139 & 0.0377 & 688,904 & 0.0342 \\
\hline $\begin{array}{l}Y_{1}^{1} \\
3\end{array}$ & $60-64$ & 1244286 & 0.054 & 671438 & 0.0334 & 572,848 & 0.0285 \\
\hline $\begin{array}{l}\text { Y1 } \\
4\end{array}$ & $65-69$ & 890340 & 0.041 & 497321 & 0.0247 & 393,019 & 0.0195 \\
\hline $\begin{array}{l}\mathrm{Y}_{1} \\
5\end{array}$ & $70-74$ & 901370 & 0.0435 & 530454 & 0.0264 & 370,916 & 0.0184 \\
\hline $\begin{array}{l}\text { Y1 } \\
6\end{array}$ & $75-79$ & 729965 & 0.0331 & 444583 & 0.0221 & 285,382 & 0.0142 \\
\hline $\begin{array}{l}\text { Y1 } \\
7\end{array}$ & $80-84$ & 462807 & 0.0206 & 292233 & 0.0145 & 170,574 & 0.0085 \\
\hline $\begin{array}{l}\mathrm{y}^{1} \\
8\end{array}$ & $85 \&>$ & 263262 & 0.0109 & 176199 & 0.0088 & 87,063 & 0.0043 \\
\hline \multicolumn{2}{|c|}{ Total: } & $\begin{array}{l}20, \quad 121, \\
641\end{array}$ & 1 & $\begin{array}{l}10, \quad 333, \\
064\end{array}$ & 0.5135 & $\begin{array}{l}9, \\
577\end{array}$ & 0.4865 \\
\hline \multicolumn{5}{|c|}{$\mathrm{S}\{w i\}$} & \multicolumn{3}{|l|}{$\Xi=\{p i\} \cup\{q i\}$} \\
\hline & & \multicolumn{2}{|c|}{$\begin{array}{l}\text { Entropy } \\
\text { (Maxim 24.2622) }\end{array}$} & $\begin{array}{l}\text { Informational } \\
\text { Energy }\end{array}$ & $\begin{array}{l}\text { Entropy } \\
\text { (Maxim 24.2622) }\end{array}$ & \multicolumn{2}{|c|}{$\begin{array}{l}\text { Informational } \\
\text { Energy }\end{array}$} \\
\hline \multicolumn{2}{|c|}{ Current (c) } & \multicolumn{2}{|c|}{4.1699} & 0.0629 & 5.0748 & \multicolumn{2}{|l|}{0.1237} \\
\hline \multicolumn{2}{|c|}{ Equal chance (ec) } & \multicolumn{2}{|l|}{4.1699} & 0.0556 & 5.1699 & \multicolumn{2}{|l|}{0.0278} \\
\hline
\end{tabular}

Table 2. The Frequencies for the Characteristics yi as for the Representative Sample Data obtained by randomly selecting data from detail reports

\begin{tabular}{|c|c|c|c|c|c|c|}
\hline Characteristics & \multicolumn{2}{|r|}{$w i$} & \multicolumn{2}{|r|}{$p i$} & \multicolumn{2}{|r|}{$q i$} \\
\hline 0 & 1 & 2 & 3 & 4 & 5 & 6 \\
\hline Y1 & 51 & 0.0448 & 23 & 0.0574 & 28 & 0.028 \\
\hline Y2 & 50 & 0.0449 & 23 & 0.0554 & 27 & 0.027 \\
\hline Y3 & 53 & 0.0487 & 25 & 0.0575 & 28 & 0.028 \\
\hline ү4 & 54 & 0.0507 & 26 & 0.0574 & 28 & 0.028 \\
\hline ү5 & 81 & 0.0721 & 37 & 0.0903 & 44 & 0.044 \\
\hline y6 & 76 & 0.0741 & 38 & 0.0781 & 38 & 0.038 \\
\hline Y7 & 81 & 0.0819 & 42 & 0.0801 & 39 & 0.039 \\
\hline y8 & 77 & 0.0741 & 38 & 0.0801 & 39 & 0.039 \\
\hline ү9 & 82 & 0.0799 & 41 & 0.0843 & 41 & 0.041 \\
\hline Y10 & 57 & 0.0585 & 30 & 0.0554 & 27 & 0.027 \\
\hline Y11 & 68 & 0.0663 & 34 & 0.0698 & 34 & 0.034 \\
\hline Y13 & 67 & 0.0682 & 35 & 0.0655 & 32 & 0.032 \\
\hline Y13 & 53 & 0.0565 & 29 & 0.0493 & 24 & 0.024 \\
\hline
\end{tabular}




\begin{tabular}{|c|c|c|c|c|c|c|c|}
\hline Y14 & 42 & 0.0507 & 26 & 0.033 & 16 & 0.016 & \\
\hline Y15 & 43 & 0.0448 & 23 & 0.0412 & 20 & 0.02 & \\
\hline ү16 & 32 & 0.0390 & 20 & 0.0246 & 12 & 0.012 & \\
\hline Y17 & 21 & 0.0273 & 14 & 0.0144 & 7 & 0.007 & \\
\hline Y18 & 12 & 0.0175 & 9 & 0.0062 & 3 & 0.003 & \\
\hline Total: & 1000 & 1 & 513 & 1 & 487 & 1 & \\
\hline & \multicolumn{3}{|c|}{$S\{w i\}$} & \multicolumn{3}{|c|}{ Informational Energy } & CW, \\
\hline & \multicolumn{2}{|c|}{$\begin{array}{l}\text { Entropy } \\
(\max 9.9657)\end{array}$} & $\begin{array}{l}\text { Informational } \\
\text { Energy }\end{array}$ & W & \multicolumn{2}{|c|}{ U } & \\
\hline Current (c) & \multicolumn{2}{|c|}{4.0627} & 0.0628 & 0.0610 & \multicolumn{2}{|c|}{0.0655} & 0.0624 \\
\hline Equal chance (ec) & 5.7958 & & 0.0556 & 0.0556 & \multicolumn{2}{|c|}{0.0556} & 0.0556 \\
\hline
\end{tabular}

Table 3. Population structure, by participation in economic activity, by age group, sex and area, in 2011

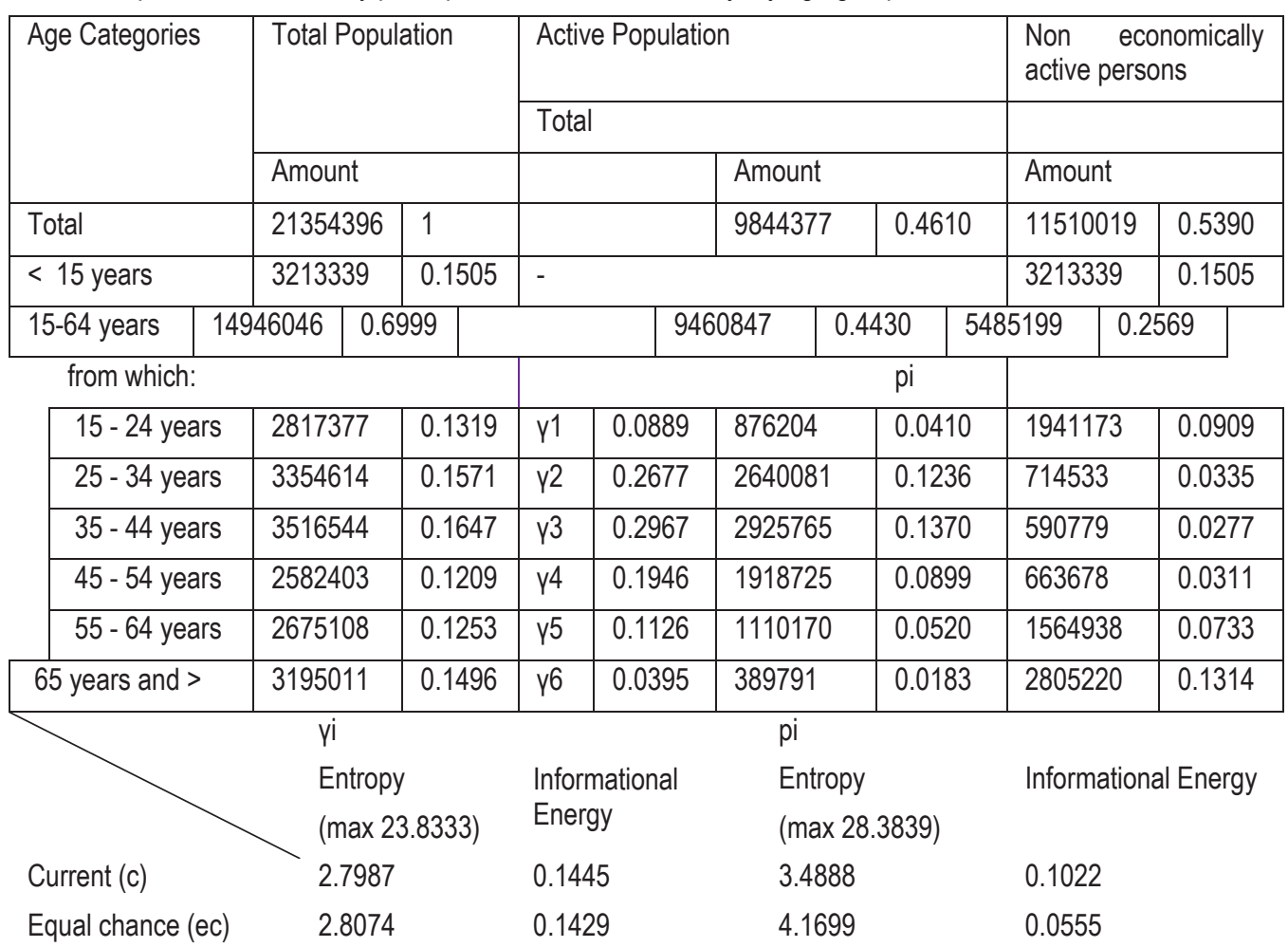

